\title{
An Iteratively Refined Circuital Model of CRLH Leaky-Wave Antennas derived from the Mushroom Structure
}

\author{
J. S. Gomez-Diaz*1, A. Alvarez-Melcon ${ }^{1}$, and T. Bertuch ${ }^{2}$ \\ ${ }^{1}$ Technical University of Cartagena, Cartagena, E-30202, Spain. \\ ${ }^{2}$ Fraunhofer FHR, 53343 Wachtberg, Germany. \\ E-mail: jsebastian_gomez@ono.com, thomas.bertuch@fhr.fraunhofer.de
}

\section{Introduction}

Metamaterial leaky-wave antennas (LWAs), which operate in their fundamental guided mode $(\nu=0)$, have grown in popularity thanks to their full-space scanning possibilities [1]. Several technologies can be employed to practically implement this type of antennas, such us microstrip, CPW, CPS or mushroom-like periodically loaded waveguides [2]. In order to analyze these structures, equivalent circuit models may be used [1]. These models are able to accurately compute the propagation constant of the structure under analysis, but have some difficulties to obtain the radiation characteristics. Usually, time-consuming full-wave simulations are required for this last step. In this paper, a novel and efficient modal-based iterative circuital model is presented for the accurate analysis of the complex propagation constant related to mushroom-like CRLH LWAs.

\section{Iterative Circuital Model of Mushroom-Like CRLH LWAs}

The topology of the proposed parallel-plate waveguide (PPW) CRLH LWA is shown in Fig. 1 (top). At the bottom, an equivalent circuit for a single unit cell section is given. The main difference of this circuit with the regular CRLH unit cell model [1] is the presence of an admittance $\left(Y_{\text {rad }}\right)$ in shunt with the series capacitor $\left(C_{L}\right)$. This admittance represents the coupling of the field inside the loaded PPW to free space via the slots in the upper metal plane. Its value depends on the physical dimensions of the antenna, on frequency and on the effective wave-number $\left(k_{\text {eff }}\right)$ related to the CRLH transmission-line (TL). Exploiting the assumption of a balanced unit cell, the other non-dispersive circuital parameters of the unit cell model may be derived using standard theory of TL metamaterials [1].

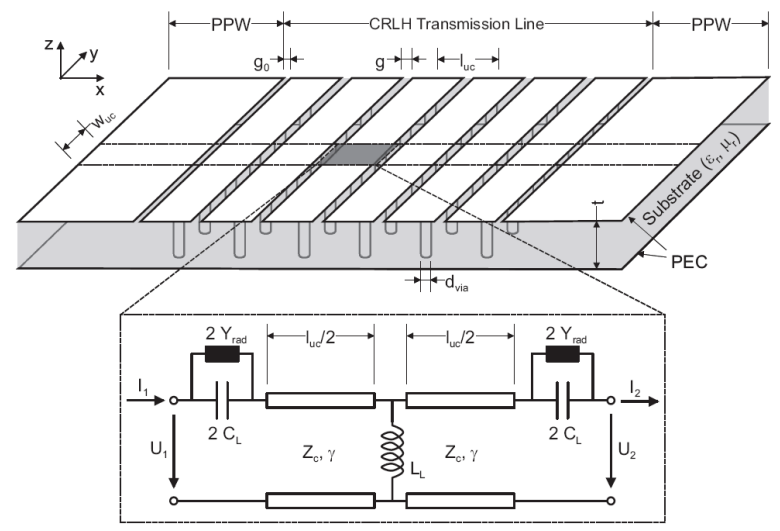

Figure 1: Topology of a CRLH LWA comprising a periodically loaded PPW (top) and its associated equivalent unit cell circuital model (bottom). 
In order to compute the dispersive radiation admittance $\left(Y_{\text {rad }}\right)$, an equivalent phased array antenna model is considered. It consists of a one-dimensional periodic array of infinitely long slots in a metal plane (see Fig. 2).

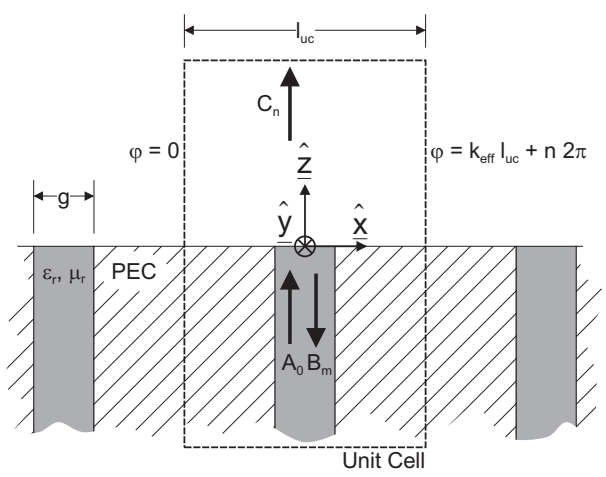

Figure 2: Cross-section of one-dimensional periodic array of infinitely long slots in a metal plane. The slots are fed by dielectrically loaded PPWs.

Using phased-array theory, we assume that all array elements are fed with the same magnitude and a progressive phase shift. Due to this, it is sufficient to consider a single unit cell (array element) with imposed periodic boundary conditions in $x$ direction as shown in Fig. 2. Moreover, the imposed phased shift at a given frequency is determined by the effective wave number on the CRLH TL as $\Delta \varphi=k_{\text {eff }} l_{u c}+n 2 \pi$. Then, the single array element is studied using a modal analysis (MA) approach [3] combined with the Floquet's theorem. Specifically, the feeding PPW is excited by the fundamental waveguide mode with complex amplitude $A_{0}$, which propagates along the guide until it reaches the air discontinuity. There, some energy is reflected back towards the waveguide, creating an infinite set of discrete modes with complex amplitudes $B_{m}$. In addition, some energy is also coupled to free space. Due to the periodic boundary conditions of the array element, the magnetic vector potential which describes this radiation may be expressed as an infinity sum of discrete modes with complex amplitudes $C_{n}$ as

$$
\vec{A}_{\text {free space }}=k_{0}^{-1} \sum_{n=-\infty}^{\infty} C_{n} e^{-j\left(k_{\text {eff }}+n \frac{2 \pi}{l_{u c}}\right) x} e^{-j k_{z, n} z} \hat{z},
$$

where $k_{0}$ is the free-space wavenumber, $n \in \mathbb{Z}, l_{u c}$ is the unit cell length, and

$$
k_{z, n}= \pm \sqrt{k_{0}^{2}-\left(k_{e f f}+n \frac{2 \pi}{l_{u c}}\right)^{2}} .
$$

Finally, the slot radiation admittance is computed from the modal amplitudes of the exciting $\left(A_{0}\right)$ and reflected $\left(B_{0}\right)$ waveguide fundamental modes, as

$$
Y_{\text {rad }}=\frac{l_{u c}}{g} \sqrt{\frac{\varepsilon_{r} \varepsilon_{0}}{\mu_{r} \mu_{0}}} \frac{1-B_{0} / A_{0}}{1+B_{0} / A_{0}} .
$$

In order to demonstrate the accuracy of the proposed MA approach, the radiation impedance of a slot (gap width equal to $10 \mathrm{~mm}$ ) placed inside the described phased array structure (unit cell length equal to $23.54 \mathrm{~mm}$ ) is shown in Fig. 3, and it is validated using full-wave data. As can be observed, excellent agreement is found. 

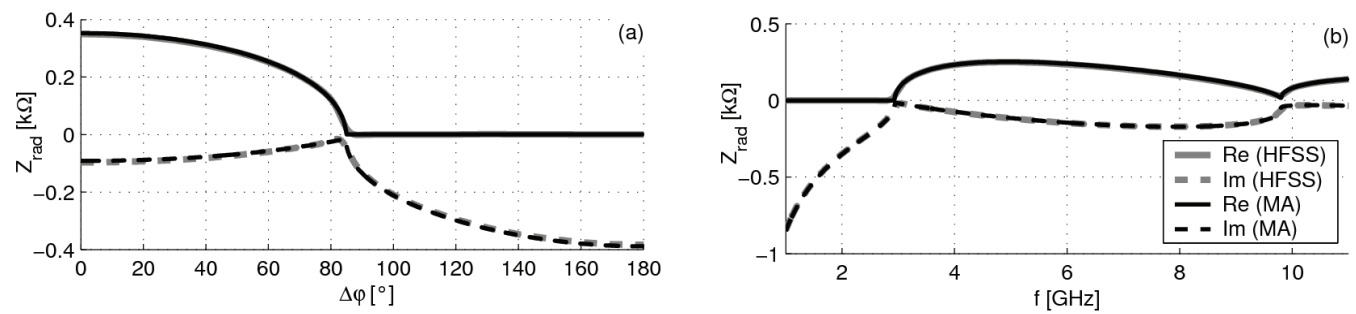

Figure 3: Comparison of radiation impedance computed by HFSS and by Modal Analysis (MA). (a) Fixed frequency $f=3 \mathrm{GHz}$. (b) Fixed phase shift between unit cell walls $\Delta \varphi=83^{\circ}$.

Since the CRLH propagation constant $\left(k_{\text {eff }}\right)$ and the slot radiation admittance $\left(Y_{\text {rad }}\right)$ are related in a complex way, an iterative scheme is proposed to determined these values for a given geometry. In an initial step, the values of $Y_{\text {rad }}$ are computed for all frequencies assuming $k_{\text {eff }}=0$. Then, the values of $k_{\text {eff }}$ are updated based on the current values of $Y_{\text {rad }}$. This procedure is repeated until convergence is reached. Numerical test have shown that this convergence is usually achieved after just 20-30 iterations.

\section{Analysis of 1D CRLH LWAs}

For the sake of validation, let us consider a unit cell with dimensions $l_{u c}=w_{u c}=$ $23.54 \mathrm{~mm}$, composed of a parallel-plate waveguide filled by a dielectric material with relative permitivity $\epsilon_{r}=1.12$ and loaded by a via-hole of diameter $d_{v i a}=1.0 \mathrm{~mm}$ and by a slot gap of width $g=0.5 \mathrm{~mm}$ (see Fig. 1). The complex propagation constant related to this unit cell is shown in Fig. 4. The real part of the propagation constant [Fig. 4 (a)] shows that the structure is balanced, with a transition frequency placed at $3.0 \mathrm{GHz}$. In addition, it can be observed that within the frequency region from 2.2 to $5.9 \mathrm{GHz}$ this magnitude is smaller than the free space wavenumber, allowing the appearance of leaky-waves. Therefore, the losses [imaginary part of the propagation constant, Fig. 4 (b)] within this frequency region are caused by leakywave radiation. Note that the proposed approach is able to accurately characterize these radiation losses, as a function of frequency and of the physical dimensions of the structure under analysis.
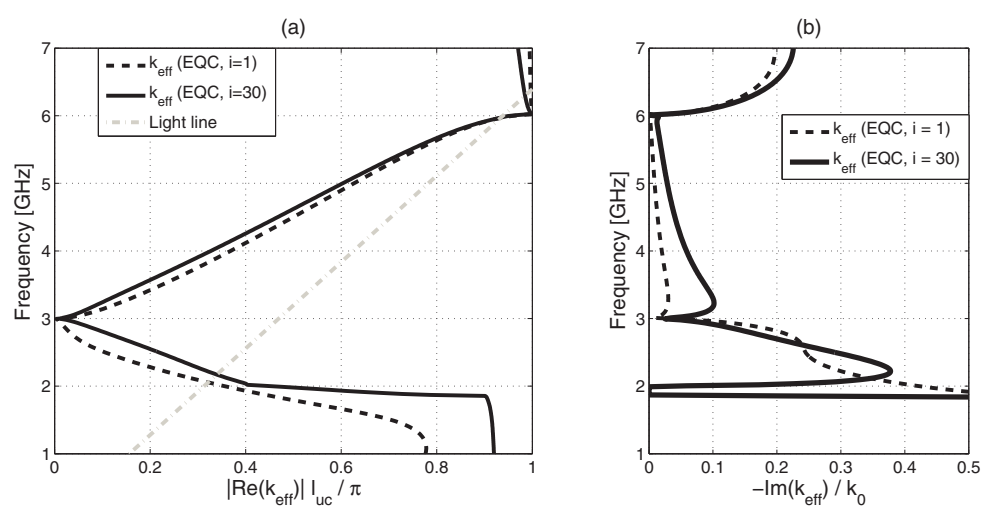

Figure 4: Dispersive behavior of the CRLH LWA under analysis, computed with the proposed iterative algorithm after $i=1$ and $i=30$ (convergence reached) iterations. (a) Brillouin diagram. (b) Attenuation (radiation) losses versus frequency. 
Finally, an antenna composed of ten unit cells is studied. For this purpose, the theory presented in the previous section is combined with an ABCD matrix approach [1]. Fig. 5 shows the scattering parameters of the structure, computed with the proposed iterative algorithm (computation time about 1.5 minutes) and validated using CST (computation time about 8 hours). As can be observed, very good agreement is achieved, demonstrating that the proposed method is able to efficiently and rigourously analyze mushroom-based CRLH LWAs, taking into account the real physical dimensions of the structure.
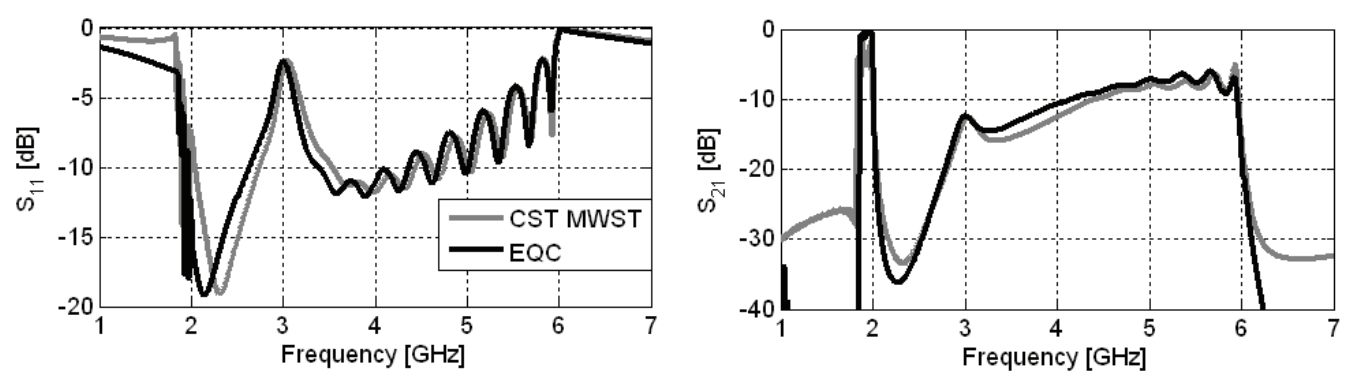

Figure 5: Comparison of scattering parameters computed by CST Microwave Studio (CST MWST) and by the proposed iterative circuital method (EQC) of a single strip CRLH LWA consisting of ten identical reference unit cells.

\section{Conclusions}

In this paper, a novel iterative algorithm has been presented for the analysis of mushroom-like PPW CRLH leaky-wave antennas. The conventional lossless CRLH unit cell configuration has been modified, including a series radiation admittance, which takes into account the coupling from the structure to free space. A phasedarray equivalent model has been introduced to compute this radiation admittance, which depends on frequency, on the physical dimensions of the structure under analysis, and on the complex propagation constant of the CRLH unit cell. Then, an iterative algorithm has been employed to determine the final values of the radiation admittance and the CRLH complex propagation constant, which are inter-related. Finally, an antenna composed of ten unit cells has been successfully analyzed with the proposed approach and validated using CST. The novel technique is accurate, several order of magnitude faster than full-wave simulations, and provides a deep insight into the physics of the antenna radiation mechanism.

\section{Acknowledgement}

This work was partially supported by the Spanish Ministry of Education and Science under Grant FPU-AP2006-015 and with the Project TEC2007-67630-C03-02.

\section{References}

[1] C. Caloz and T. Itoh, "Electromagnetic Metamaterials: Transmission Line Theory and Microwave Applications," Hoboken, Wiley and IEEE Press, 2005.

[2] T. Bertuch, "A TM leaky-wave antenna comprising a texture surface", in Proc. Int. Conf. Electromag. Adv. Appl. (ICEAA), Sept. 2007.

[3] N. Marcuvitz, "Waveguide Handbook", New York, McGraw-Hill, 1951. 